\title{
Vigilância Nutricional em Adultos: Experiência de uma Unidade de Saúde Atendendo População Favelada
}

\section{Nutritional Surveillance of Adults: The Experience of a Health Service for an Urban Slum Population}

Luiz A. dos Anjos '; Denise O. e Silva';

Simone A. Serrão ${ }^{2}$ \& Cláudia V. C. da Silva ${ }^{2}$

ANJOS, L. A. dos; SILVA, D. O.; SERRÃO, S. A. \& SILVA, C. V. C. da Nutritional

Surveillance of Adults: The Experience of a Health Service for an Urban Slum Population. Cad. Saúde Públ., Rio de Janeiro, 8 (1): 50-56, jan/mar, 1992.

The nutritional surveillance team of the Centro de Saúde Escola Germano Sinval Faria (CSEGSF) collects anthropometric (body mass and stature) as well as socioeconomic and morbidity data of low-income adults (age $\geq 20$ years) from seven slums in Manguinhos, Rio de Janeiro. This report presents data on 1352 people (1111 women and 241 men) seen between August and December 1989. Nutritional status (NS) was determined according to the value of body mass index $\left(B M I=K g \cdot\left(m^{2}\right)^{-1}\right)$ : underweight $(U W ; B M I<20)$, normal $(20 \leq B M I<25)$, overweight $(O W ; 25 \leq B M<30)$ and obesity $(B M I \geq 30)$. The frequency (\%) of NS was: $U W=9.5$ e 17.5; normal $=37.2$ and 46.9; $O W=31.3$ e 29.0; obesity $=22.0$ e 6.6 for women and men, respectively. These data indicate that among these low-income women at least 50\% are $O W$. The authors speculate that this high frequency of $O W$ may be associated not only with dietary intake (quality and quantity) but also with the number of pregnancies and maybe an adaptation to low energy intake during infancy. As far as the service is concerned, a nomogram to calculate BMI was introduced in the routine to be used by the professionals of the PAISA (Adult Health Program).

Keywords: Health Service; Nutritional Surveillance; Obesity; Health of Adults

\section{INTRODUÇÃO}

O Sistema de Vigilância Alimentar e Nutricional (SISVAN) tem como um de seus objetivos principais o controle permanente do quadro epidemiológico da situação nutricional da população como um todo ou de segmentos mais vulneráveis dessa população. Um dos vários problemas do SISVAN está relacionado com a escolha do indicador a ser utilizado na avaliação nutricional. Existe uma concordância

\footnotetext{
' Centro de Estudos da Saúde do Trabalhador e Ecologia Humana da Escola Nacional de Saúde Pública. Rua Leopoldo Bulhões, 1480, Rio de Janeiro, RJ, 21041 , Brasil.

${ }^{2}$ Centro de Saúde Escola Germano Sinval Faria da Escola Nacional de Saúde Pública. Rua Leopoldo Bulhöes, 1480, Rio de Janeiro, RJ, 21041, Brasil.
}

entre pesquisadores na utilização do crescimento físico como indicador de saúde e nutrição em crianças (Monteiro, 1984; Waterlow et al., 1977). Para estudos epidemiológicos, parece que o índice de massa corporal (IMC) pode ser utilizado como indicador do estado nutricional (EN) em adultos (Smalley et al., 1990), pela facilidade de obtenção dos dados e pelo poder de predição do $\mathrm{MCC}$ da morbi-mortalidade encontrada em estudos longitudinais (Hoffmans et al., 1988; Waaler, 1988). Na atualidade, não se conhece a distribuição do IMC na população brasileira. O presente trabalho apresenta dados relativos à avaliaçāo do $\mathrm{EN}$, utilizando o $\mathrm{IMC}$, da demanda espontânea no Centro de Saúde Escola Germano Sinval Faria (CSEGSF), dentro da proposta de implantação do SISVAN do adulto no serviço. 


\section{MATERIAIS E MÉTODOS}

A amostra desse trabalho constitui-se de 1.352 indivíduos (1.111 mulheres e 241 homens), maiores de 20 anos de idade, atendidos entre agosto e dezembro de 1989 no Centro de Saúde Escola Germano Sinval Faria (CSEGSF), que é uma unidade de treinamento ligada à Escola Nacional de Saúde Pública, da Fundação Oswaldo Cruz, no Rio de Janeiro. A população atendida no CSEGSF compõe-se de moradores de sete favelas e de um conjunto habitacional da região de Manguinhos.

Todos os dados foram obtidos dos prontuários médicos dos pacientes atendidos. As medidas antropométricas (massa corporal, MC e estatura, EST) são realizadas pelos profissionais treinados do setor de enfermagem. A MC é medida com a pessoa vestida, numa balança Filizola, com precisão de $0,1 \mathrm{~kg}$. A EST é medida no estadiômetro da própria balança, com precisão de $0,1 \mathrm{~cm}$. Para esse trabalho e na rotina do serviço, os dados de MC e EST são utilizados para o cálculo do índice de Massa Corporal ( $\left.\mathbb{M} C=\mathrm{Kg}\left(\mathrm{m}^{2}\right)^{-1}\right)$, indicando o estado nutricional (EN) como baixo peso (BP; IMC $<20$ ), Normal $(20 \leq \mathrm{IMC}<25)$, Sobre-Peso (SP; $25 \leq \mathrm{IMC}<30$ ) e Obeso (IMC $\geq 30$ ) (Bray, 1989). Além dos dados antropométricos, a idade e o sexo dos pacientes foram obtidos dos prontuários médicos.

Os dados foram transferidos para um microcomputador $e$ a análise estatística foi realizada através do programa estatístico SAS (SAS, 1985). Os dados foram estratificados por sexo, faixa etária e estado nutricional, e foram obtidas as médias e desvios padrão das medidas antropométricas. A influência do sexo nas medidas antropométricas foi avaliada pela análise de covariância, utilizando-se a idade como covariável. Coeficientes de correlação Pearson foram gerados entre os valores de IMC, massa corporal e estatura para toda a amostra e por faixa etária e sexo. $O$ valor de $\mathrm{p}=0,05$ foi utilizado para determinar a significância estatística.

\section{RESULTADOS}

Os dados antropométricos por faixa etária e sexo são apresentados na Tabela 1. A primeira observação a se fazer é a maior quantidade de atendimentos a pacientes do sexo feminino, $o$ que demonstra a caracteristica de a Unidade de Saúde ter uma maior demanda materno-infantil. Os valores da massa corporal e da estatura das mulheres foram sistematicamente inferiores aos dos homens, em todas as faixas etárias. Entretanto, o IMC foi superior para as mulheres de todas as idades. A influência do sexo foi significativa para todas as medidas antropométricas, controlando-se pela idade.

A frequêencia (\%) do estado nutricional (EN) observada é apresentada na Figura 1. Os homens apresentam um percentual maior de baixo peso, enquanto as mulheres apresentam maior grau de obesidade. No total, pelo menos $50 \%$ das mulheres apresentaram sobrepeso (SP).

As médias do IMC em função da idade e sexo estão representadas graficamente na Figura 2. Os valores médios do IMC das mulheres ultrapassam o ponto de corte de SP (IMC $\geq 25$ ) na faixa entre 30 e 40 anos. Os dados dos homens ficam abaixo desse limite para todas as faixas, etárias com exceção dos $>70$ anos cujo " $n$ " foi igual a 23 (Tabela 1).

\section{DISCUSSÃO}

Para um sistema de vigilância nutricional, é fundamental que se utilizem indicadores fáceis de se obter e que sejam válidos para a avaliação do estado nutricional (EN). Em crianças, utiliza-se o crescimento como indicador do EN. Para adultos, é necessário que se desenvolva metodologia simples da avaliação da composiçāo corporal (Lukaski, 1987), já que não se pode utilizar o crescimento físico. Comparações das dimensões corporais, como massa corporal e, principalmente, estatura, com padrões internacionais também não devem ser feitas, já que a estatura do adulto sofre influência genética importante, que não se consegue distinguir dos outros fatores ambientais. $\mathrm{Na}$ ausência de metodologia de avaliação da composição corporal, o índice de massa corporal (IMC) é utilizado por ter alta correlação com a massa corporal e gordura corporal e baixa correlação com a estatura (Cronk \& Roche, 
1982; Womersley \& Durnin, 1977; Micozzi et al., 1986). Apesar do IMC ser preconizado como indicador do estado nutricional de adultos (WHO, 1985), ainda não se tem clareza sobre os pontos de corte de normalidade em relação ao sexo e idade (Bray, 1989), principalmente em populaçōes de paises do Terceiro Mundo (Norgan, 1990). Com vistas a facilitar a implementação da Vigilância Nutricional do adulto no CSEGSF e até que se tenha maiores informações, achamos por bem utilizar um valor único ( $\mathrm{MMC} \geq 25$ ) como critério para sobrepeso, para ambos os sexos e para todas as faixas etárias, como tem sido sugerido na literatura (Anônimo, 1985; Bray, 1989). Vale ressaltar que o IMC não mede composição corporal e, portanto, deve-se ter cuidado ao diagnosticar "obesidade" como fazemos para o $\mathrm{IMC} \geq 30$.

Como demonstrado para outras populações, o IMC apresentou alta correlação com a massa corporal ( $\mathbf{r}=0,91$ para a amostra toda e sempre acima de 0,88 para as várias faixas etárias e sexo) e fraca correlação com a estatura, apesar de significativa em alguns casos ( $r$ geralmente abaixo de 0,10 ). Parece, portanto, que o IMC pode ser utilizado na presente amostra. Houve uma alta freqüência de sobrepeso, principalmente nas mulheres. A pergunta que se faz é como pode uma população de baixa renda apresentar sobrepeso. Apesar de não termos dados suficientes para a explicação dos fatores determinantes do sobrepeso nessa população e a presente amostra ser constituída de individuos que procuraram o serviço médico do CSEGSF, o que poderia não apresentar a freqüência de sobrepeso da população favelada de Manguinhos, podemos especular algumas coisas. A primeira e mais óbvia explicação passa por fatores alimentares quantitativos e qualitativos, apesar da quase sempre fraca correlação entre adiposidade e ingestão alimentar descrita na literatura (Rolland-Cachera \& Bellisle, 1986). É de se imaginar que, para essa população, a ingestão protéica seja baixa e a

TABELA 1. Valores Antropométricos (Médias, $\overline{\mathrm{X}}$, e Desvio Padrão, DP) de 1.352 Indivíduos Atendidos, entre Agosto e Dezembro de 1989, no CSEGSF, em Função do Sexo e Idade, e os Valores de "F" da Análise de Variância da Influência do Sexo na Massa Corporal, Estatura e Indice de Massa Corporal, Controlando-se pela Idade

\begin{tabular}{|c|c|c|c|c|c|c|c|c|}
\hline \multirow[t]{2}{*}{$\begin{array}{l}\text { Faixa } \\
\text { Etária }\end{array}$} & \multirow[t]{2}{*}{ Sexo } & \multirow[t]{2}{*}{$\mathrm{n}$} & \multicolumn{2}{|c|}{$\begin{array}{c}\text { Massa } \\
(\mathrm{Kg})\end{array}$} & \multicolumn{2}{|c|}{$\begin{array}{l}\text { Estatura } \\
(\mathrm{cm})\end{array}$} & \multicolumn{2}{|c|}{$\begin{array}{l}\text { Índice de Massa Corporal } \\
\left(\mathrm{K}_{\mathrm{g} .}\left(\mathrm{m}^{2}\right)^{-1}\right)\end{array}$} \\
\hline & & & $\overline{\mathrm{x}}$ & $\mathrm{DP}$ & $\overline{\mathrm{x}}$ & $\mathrm{DP}$ & $\overline{\bar{x}}$ & $\mathrm{DP}$ \\
\hline \multirow[t]{2}{*}{$20 \cdot 29,9$} & F & 290 & 58,2 & 11,2 & 155,8 & 6,8 & 24,0 & 4,5 \\
\hline & $\mathbf{M}$ & 58 & 64,6 & 09,4 & 168,0 & 8,3 & 22,9 & 3,2 \\
\hline \multirow[t]{2}{*}{$30-39,9$} & F & 255 & 60,6 & 12,0 & 154,9 & 5,9 & 25,3 & 4,9 \\
\hline & $\mathbf{M}$ & 44 & 64,3 & 11,2 & 164,2 & 6,4 & 23,8 & 3,7 \\
\hline \multirow[t]{2}{*}{$40-49,9$} & $F$ & 175 & 65,4 & 16,4 & 153,7 & 6,8 & 27,6 & 6,3 \\
\hline & $\mathbf{M}$ & 31 & 67,5 & 14,3 & 166,1 & 6,5 & 24,4 & 4,7 \\
\hline \multirow[t]{2}{*}{$50-59,9$} & $F$ & 173 & 66,7 & 15,0 & 152,9 & 6,0 & 28,5 & 5,9 \\
\hline & $\mathbf{M}$ & 47 & 66,6 & 12,8 & 165,3 & 7,4 & 24,4 & 4,2 \\
\hline \multirow[t]{2}{*}{$60-69,9$} & $\mathbf{F}$ & 157 & 62,3 & 13,4 & 150,5 & 6,5 & 27,4 & 5,2 \\
\hline & $\mathbf{M}$ & 38 & 64,6 & 10,5 & 163,8 & 5,8 & 24,1 & 3,8 \\
\hline \multirow[t]{2}{*}{$>70$} & $F$ & 61 & 59,7 & 12,9 & 148,0 & 6,0 & 27,2 & 5,6 \\
\hline & $\mathbf{M}$ & 23 & 68,1 & 13,4 & 163,7 & 6,7 & 25,3 & 4,2 \\
\hline Influência & & & \multicolumn{2}{|c|}{$16,39^{*}$} & \multicolumn{2}{|c|}{$658,2^{*}$} & \multicolumn{2}{|c|}{$38,1^{*}$} \\
\hline
\end{tabular}

- Valores de " $F^{*}$ da análise de covariância da influência do sexo nas medidas antropométricas, controlando-se pela idade. 
FIGURA 1. Freqüência (\%) do Estado Nutricional, Dado pelo Índice de Massa Corporal (IMC) em Função do Sexo de 1.352 Indivíduos (1.111 Mulheres e 241 Homens) Atendidos no CSEGSF, entre Agosto e Dezembro de 1989

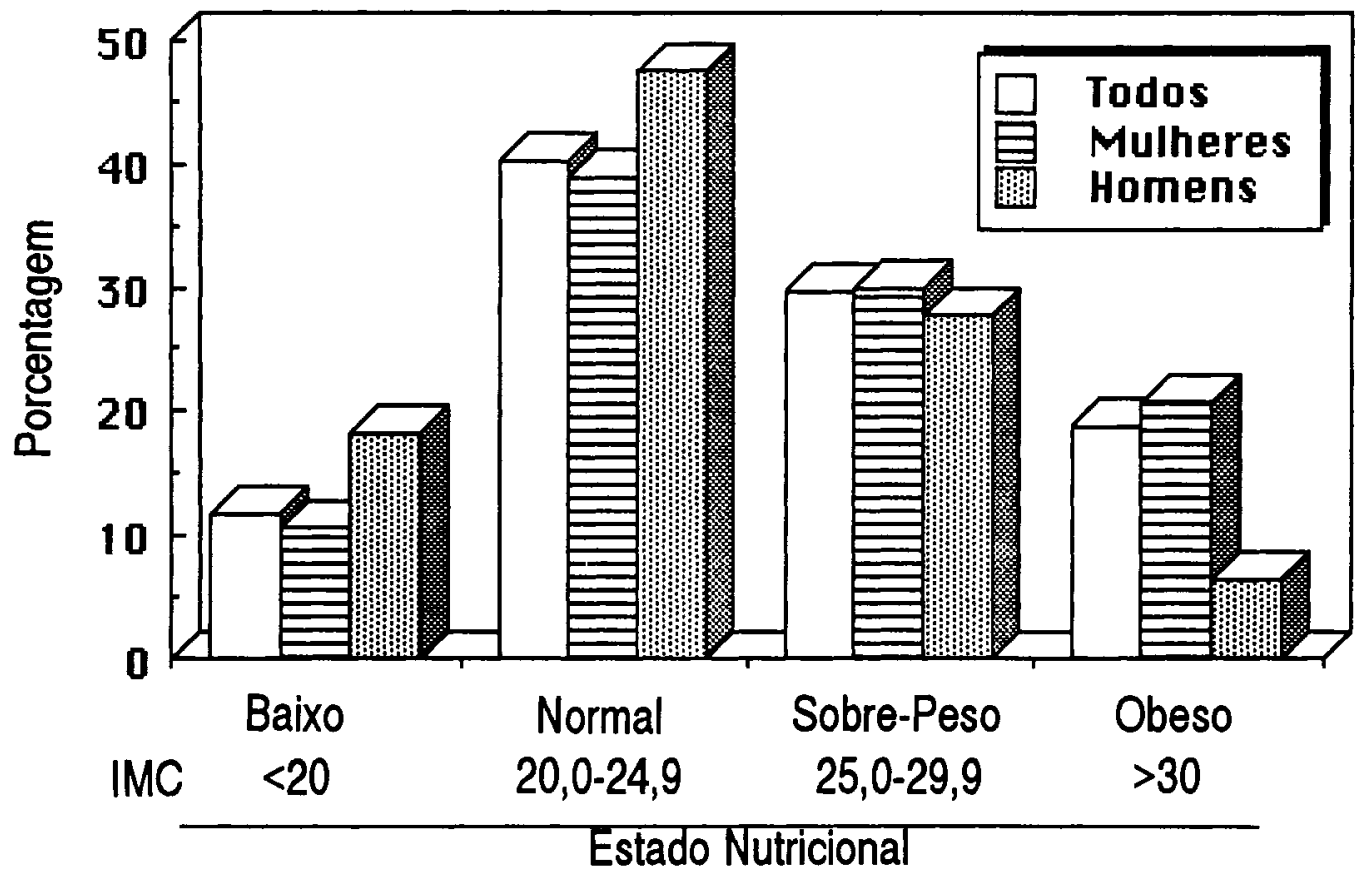

FIGURA 2. Médias e Erro Padrão da Média do Índice de Massa Corporal em Função das Faixas Etárias de 1.111 Mulheres e 241 Homens Atendidos no CSEGSF, entre Agosto e Dezembro de 1989

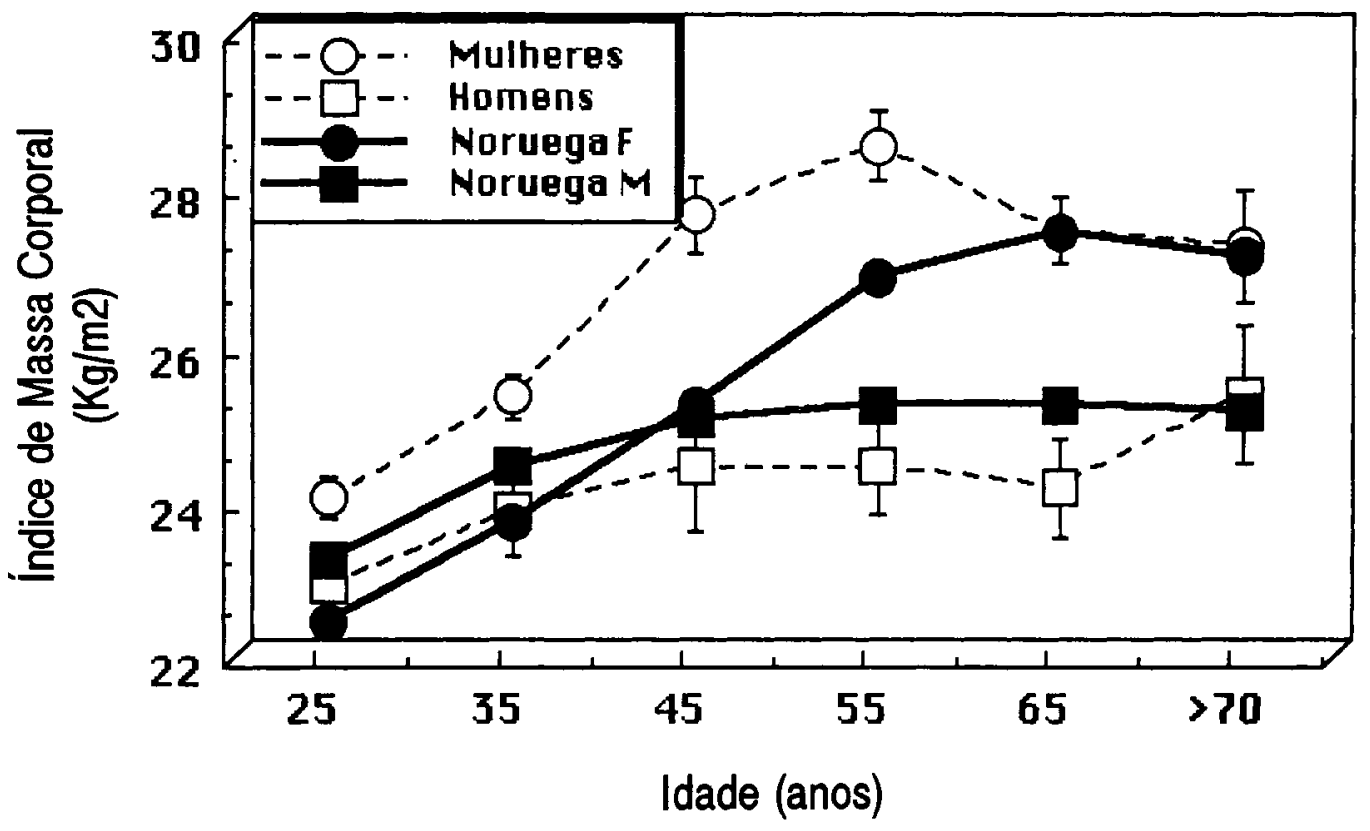


ingestão de alimentos ricos em hidratos de carbono e gordura seja alta. Essa dieta poderia, sem suprir a necessidade energética ao organismo, fornecer gordura para ser depositada. Associada aos fatores alimentares, é possivel que ocorra uma adaptação físiológica à baixa ingestão energética nas primeiras décadas de vida, levando à desnutrição na infância e ao excesso de peso na vida adulta. Existem dados ainda não conclusivos que apontam que as meninas são mais resistentes à desnutrição por estresse ambiental do que os meninos (Engle \& Levin, 1984). Se verdade ou não, esta diferença na "adaptação nutricional" ao estresse ambiental poderia explicar o fato de mulheres americanas de baixa renda e/ou menor nível educacional terem maior gordura corporal, medida através das dobras cutâneas ou o $\mathrm{MMC}$, do que mulheres americanas de renda mais alta e/ou maior nível educacional (Flegal et al., 1988; Garn et al., 1981). Dados mais recentes em população "hispânica" americana (descendentes de cubanos, porto-riquenhos e mexicanos), dita de condiçōes sócio-econômicas menos privilegiadas, mostram semelhança do IMC entre os sexos até os 17 anos de vida, mas as mulheres adultas apresentam valores de IMC sistematicamente maiores do que os homens (Fanelli-Kuczmarski \& Woteki, 1990), como foi o caso na amostra de Manguinhos.
Outro fator associado ao sexo é a gravidez. É sabida a dificuldade de retorno da massa corporal pré-gravidez, principalmente em múltiplas gravidezes.

É de se supor que os homens tendam a ter um gasto energético maior do que as mulheres, principalmente associado às atividades de trabalho. Por todos esses motivos, 0 achado de sobrepeso em mulheres e de baixo peso em homens que se consultaram no serviço não é simples de ser explicado, e estudos mais complexos, com uma abordagem interdisciplinar, devem ser realizados para se explicar o aparente paradoxo de se observar sobrepeso nas mulheres de baixa renda.

Ainda não se conhece a distribuição do indice de massa corporal (IMC) na população brasileira como um todo ou em seus segmentos. Os dados do presente trabalho foram comparados com dados de uma amostra grande ( $\mathrm{n}=1,7$ milhões) da população norueguesa (Waaler, 1988) e são apresentados na Figura 3. Estes dados foram utilizados por constituírem a maior amostra do conhecimento dos autores na literatura. Entretanto, deve-se ter muita cautela nessa comparação, já que as duas amostras são bastante diferentes entre si. De qualquer jeito, pode-se observar a semelhança das curvas médias das duas amostras, sendo que a curva das mulheres norueguesas só.

FIGURA 3. Médias e Erro Padrão da Média do Índice de Massa Corporal em Função das Faixas Etárias de 1.111 Mulheres e 24I Homens Atendidos no CSEGSF, entre Agosto e Dezembro de 1989, em Relação a Dados da Literatura Norueguesa (Waaler, 1988)

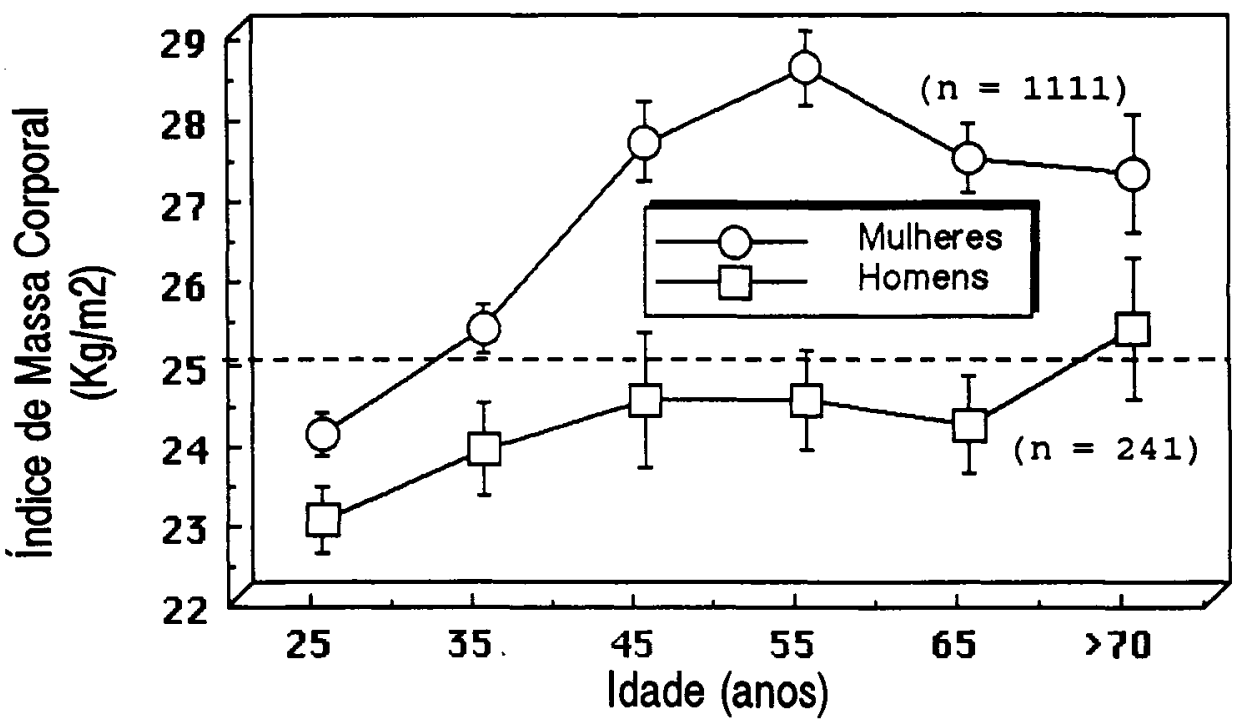


ultrapassa o ponto de corte de sobrepeso na faixa etária de 40 a 50 anos, sendo a curva dos homens brasileiros sempre inferior ao da amostra norueguesa. Ou seja, as mulheres de Manguinhos alcançam, em média, o nível de sobrepeso mais precocemente que as mulheres norueguesas, e os homens de Manguinhos apresentam menor massa corporal em função da estatura do que os noruegueses. $O$ interessante é que, em geral, o "padrão" de curva do IMC em função da idade é bem semelhante, o que nos leva a interrogar a possibilidade desse "padrão" de curva ser independente das condições ambientais. Além das condições precárias as quais essas mulheres estão submetidas, o achado de sobrepeso, com todas as suas conseqüências sobre a saúde $\mathrm{e}$ as relações afetivas, sociais e psicológicas, demonstra o quadro perverso da desigualdade social na nossa população.

Do ponto de vista de serviço, o projeto de implantação da vigilância nutricional do adulto trouxe de positivo a proposta de utilização de um nomograma de cálculo do IMC que já é usado pelos profissionais do Programa de Atenção Integral a Saúde do Adulto, no CSEGSF, como, aliás, é recomendado por instituiçōes médicas internacionais (NIH, 1985). Os resultados aqui apresentados estão sendo discutidos no Programa, com vistas a futuras intervençōes, a nível individual e coletivo, na população favelada de Manguinhos.

\section{AGRADECIMENTOS}

A presente pesquisa foi financiada em parte pelo CNPq, processos números 301076/90-8 e $503069 / 88-4$. Os autores gostariam de agradecer aos dois revisores anônimos pelas valiosas sugestōes.

\section{RESUMO}

ANJOS, L. A. dos; SILVA, D. O.; SERRÃO, S. A. \& SILVA, C. V. C. da Vigilância Nutricional em Adultos: Experiência de uma Unidade de Saúde Atendendo População Favelada. Cad. Saúde Públ., Rio de Janeiro, 8 (1): 50-56, jan/abr, 1992.

$O$ sistema de vigilância nutricional de adultos (idade $\geq 20$ anos), realizado no Centro de Saúde Escola Germano Sinval Faria (CSEGSF), registra diariamente dados de massa corporal (kg), estatura (m) e morbidades, além de dados sócio-econômicos de sua clientela, que são moradores de sete favelas da região de Manguinhos, RJ. Esse sistema tem como objetivo o controle permanente, a níveis individual e epidemiológico, da situação nutricional desta população. O presente trabalho apresenta dados relativos à demanda espontânea de 1.352 individuos (1.111 mulheres e 241 homens) maiores de 20 anos de idade atendidos entre agosto e dezembro de 1989. $O$ estado nutricional (EN) foi determinado a partir do índice de massa corporal (IMC = kg. $\left.\left(\mathrm{m}^{2}\right)^{-1}\right)$ : baixo peso (BP; IMC $<20$ ), normal $(20 \leq \mathrm{IMC}<25)$, sobrepeso (SP; $25 \leq \mathrm{MMC}<30$ ) e obeso ( $\mathrm{MCC} \geq 30$ ). A frequiência (\%) do EN para mulheres e homens, respectivamente, foi: $\mathrm{BP}=9,5 \mathrm{e}$ 17,5 ; normal $=37,2$ e 46,$9 ; \mathrm{SP}=31,3$ e 29,0 ; obesidade $=22,0$ e 6,6. Estes dados demonstram que, nesta população ambulatorial de baixa renda, pelo menos $50 \%$ das mulheres apresentam SP. Os autores especulam que essa alta freqüência de SP pode estar relacionada com fatores alimentares, qualitativos e quantitativos, além $\mathrm{da}$ influência do número de gravidezes, $\mathrm{e}$ mais remotamente, a possibilidade de haver adaptação à baixa ingestão energética na infância. Com relação ao serviço, houve uma proposta de utilização de um nomograma para o cálculo do IMC pelos profissionais do Paisa (Programa de Atenção Integral a Saúde do Adulto), o que facilitará a implantação da vigilância nutricional no serviço.

Palavras-Chave: Serviço de Saúde; Avaliação Nutricional; Obesidade; Saúde do Adulto 


\section{REFERÊNCIAS BIBLIOGRÁFICAS}

ANONIMO, 1989. Diet and health: Scientific concepts and principles. Nutrition Reviews, 45: 276287.

ANÓNIMO, 1989. National Academy of Sciences Report on Diet and Health. Nutrition Reviews, 47: $142-149$.

BRAY, G. A., 1989. Classification and evaluation of the obesities. Medical Clinics of North America, 73: 161-184.

CRONCK, C. E. \& ROCHE, A. F., 1982. Race and sex-specific reference data for triceps and subscapular skinfolds and weight/stature ${ }^{2}$. American Journal of Clinical Nutrition, 35: 347354.

ENGLE, P. L. \& LEVIN, R. J., 1984. Sex differences in the effects of malnutrition on mental development: A review and some hypothesis. In: Malnutrition and Behavior: Critical Assessment of Key lssues (J. Brozek \& B. Schuch, eds.), pp. 396-410. Lausanne, Switzerland: Nestlé Foundation.

FANELLI-KWCZMARSKI, M. \& WOTEKI, C. E., 1990. Monitoring the nutritional status of the hispanic population: Selected findings for Mexican Americans, Cubans and Puerto Ricans. Nutrition Today, 25: 6-11.

FLEGAL, K. M.; HARLAN, W. R. \& LANDES, J. R., 1988. Secular trends in body mass index and skinfold thickness with socioeconomic factors in young adult women. American Journal Clinical Nutrition, 48: 535-543.

GARN, S. M.; HOPKINS, P. J. \& RYAN, A. S., 1981. Differential fatness gain of low income boys and girls. American Journal of Clinical Nutrition, 34: 1465-1468.

HOFFMANS, M. D. A.; KROMHOUT, D. \& LEZENNE-COULANDER, C., 1989. Body mass index at the age of 18 and its effects on 32 year-mortality from coronary heart and cancer. A nested case-control study among the entire 1932 Dutch Male Birth Cohort. Journal of Clinical Epidemiology, 42: 513-520.

LUKASKI, H. C., 1987. Methods for the assessment of human body composition: Traditional and new. American Journal of Clinical Nutrition, 46: 537-556.

MICOZZI, M. S.; ALBANES, D., JONES, D. Y. \& CHUMLEA, W. C., 1986. Correlations of body mass indices with weight, stature, and body composition in men and women in NHANES I and II. American Journal of Clinical Nutrition, 44: 725-731.
MONTEIRO, C. A., 1984. Critérios antropométricos no diagnóstico da desnutrição em programas de assistência à criança. Revista de Saúde Pública, 18: 209-217.

NIH, 1985. Health implications of obesity. National Institute of Health Consensus Development Conference Statement. Annals of Internal Medicine, 103: 1073-1077.

NORGAN, N. G., 1990. Body mass index and body energy stores in developing countries. European Journal of Clinical Nutrition, 44 (suppl. 1): 79 . 84.

ROLLAND-CACHERA, M. F. \& BELLISLE, F., 1986. No correlation between adiposity and food intake: Why are working class children fatter? American Journal of Clinical Nutrition, 44: 779787.

SAS - SAS, 1985. User's guide: Statistics, version 5 Edition. Cary, NC: SAS Institute.

SMALLEY, K. J.; KNERR, A. N.; KENDRICK, Z. V., COLLIVER, J. A. \& OWEN, O. E., 1990. Reassessment of body mass indices. American Journal of clinical Nutrition, 52: 405-408.

WAALER, H. T., 1988. Hazard of obesity - the Norweigian experience. Acta Medica Scandinavica, 713 (Suppl.): 17-21.

WATERLOW, J. C.; BUZINA, R.; KELLER, W.; LANE, J. M.; NICHAMAN, N. Z. \& TANNER, J. M., 1977. The presentation and use of height and weight data for comparing the nutritional status of groups of children under the age of 10 years. Bulletin of the World Health Organization, 55: 489-498.

WOMERSLEY, J. \& DURNIN, J. V. G. A., 1977. A comparison of the skinfold method with extent of "overweight" and various weight-height relationships in the assessment of obesity. British Journal of Nutrition, 38: 271-282.

WHO (WORLD HEALTH ORGANIZATION), 1985. Energy and protein requirements. Report of a joint $\mathrm{FAO} / \mathrm{WHO} / \mathrm{UNU}$ meeting. WHO Technical Report series, n² 724, Geneva: WHO. 